\title{
Neither high-dose nor low-dose brachytherapy increases flap morbidity in salvage treatment of recurrent head and neck cancer
}

\author{
Peter W. Henderson, MD, MBA', David I. Kutler, MD², Bhupesh Parashar, MD³, David M. Otterburn, MD', Marc A. Cohen, MD². \\ Jason A. Spector, MD FACSl, 2 \\ 'Division of Plastic Surgery, Department of Surgery, ${ }^{2}$ Department of Otolaryngology, ${ }^{3}$ Department of Radiation Oncology, \\ New York - Presbyterian/Weill Cornell Medical Center, New York, NY, USA
}

\begin{abstract}
Purpose: While brachytherapy is often used concurrently with flap reconstruction following surgical ablation for head and neck cancer, it remains unclear whether it increases morbidity in the particularly high risk subset of patients undergoing salvage treatment for recurrent head and neck cancer (RH\&NC).

Material and methods: A retrospective chart review was undertaken that evaluated patients with RH\&NC who underwent flap coverage after surgical re-resection and concomitant brachytherapy. The primary endpoint was flap viability, and the secondary endpoints were flap and recipient site complications.

Results: In the 23 subjects included in series, flap viability and skin graft take was $100 \%$. Overall recipient site complication rate was 34.8\%, high-dose radiation (HDR) group 50\%, and low-dose radiation (LDR) group $29.4 \%$. There was no statistically significant difference between these groups.

Conclusions: In patients who undergo flap reconstruction and immediate postoperative radiotherapy following salvage procedures for RH\&NC, flap coverage of defects in combination with brachytherapy remains a safe and effective means of providing stable soft tissue coverage.
\end{abstract}

J Contemp Brachytherapy 2016; 8, 4: 308-312

DOI: $10.5114 /$ jcb.2016.61976

Key words: brachytherapy, flap reconstruction, head and neck cancer, HDR, LDR.

\section{Purpose}

Despite multi-modality therapy, cancer of the head and neck region carries recurrence rates as high as $50 \%$, depending on the initial stage [1,2]. Available treatment modalities include, either alone or in combination, chemotherapy, surgical resection, and, less commonly, radiation. As many patients have already been treated with a full radiation dose, treatment with external beam radiation is either contraindicated, or at least requires special techniques. Although localized delivery of high-dose radiation remains a viable option in appropriately selected patients, the attendant soft tissue deficits in the area of previous resection, combined with the poor wound healing inherent to previously radiated tissues make the likelihood of wound healing complications significantly greater (satisfactory perioperative outcomes are especially important in the neck, where poor wound healing can lead to exposure of vital structures, fistula, carotid blowout, and also the risk of death).

Brachytherapy is delivered either via removable catheters secured to the area of surgical resection through which radioisotopes are inserted (often referred to as high-dose radiation, or HDR), or with permanent radioisotope-containing seeds placed into the wound bed at the time of surgical resection (often referred to as lowdose radiation, or LDR). As external beam radiation (EBR) alone or in combination with surgery and chemotherapy, is a first line treatment for nearly all head and neck cancer, most patients with recurrence are not candidates to receive further EBR. Brachytherapy can mitigate more global toxicity associated with EBR by allowing precise targeting of the radiation to a limited area (often less than $1 \mathrm{~cm}$ in radius), making many patients with local recurrence candidates for surgical resection [3]. Because re-resection usually involves previously radiated tissues, the likelihood of wound healing complications becomes significant unless vascularized flaps are utilized in the reconstruction. Historically, there has been some hesitation to perform concurrent flap coverage and brachytherapy because of concern regarding the toxic effects of the directly adjacent radiation to the newly manipulated tissue $[4,5]$.
Address for correspondence: Jason A. Spector, MD, FACS, Division of Plastic Surgery, Department of Surgery, New York - Presbyterian/Weill Cornell, Medical Center, 525 East 68th Street, Payson 709A, New York 10065, USA, phone: +1 212746 4532, fax: +1 212746 8592, ఐ e-mail: jas2037@med.cornell.edu
Received: 12.06 .2016 Accepted: 10.08 .2016 Published: 24.08 .2016 
The safety and potential for morbidity of flap reconstruction and brachytherapy has been studied in the past, but the cohort of particularly high risk patients with recurrent head and neck cancer have not yet been specifically evaluated, nor has the relative safety of HDR versus LDR been compared. This leaves an important gap in our understanding of how best to treat this difficult clinical scenario. Therefore, the purpose of this study was to compare the safety and efficacy of flap coverage of surgical wounds in patients with RH\&NC who concurrently undergo either HDR or LDR.

\section{Material and methods}

A retrospective chart review was performed of patients between 2007 and 2016 with recurrent head and neck cancer with metastases in the cervical lymph node field who underwent surgical treatment and concurrent brachytherapy. The head and neck surgery team performed the surgical ablation, which involved resection of the recurrent mass, as well as in almost every case some form of cervical lymphadenectomy (either selective neck dissection, modified radial neck dissection, or radical neck dissection). In order to minimize exposure of the surgical team to the brachytherapy radiation, the plastic surgery team would then elevate their chosen flap prior to assisting the radiation oncologist in precise placement of the HDR catheters or LDR mesh/beads (Figure 1). High-dose radiation was delivered via temporary implantation of catheters that delivered ${ }^{192}$ Ir for 4-6 days and then removed at the bedside, and LDR was delivered via permanent ${ }^{131} \mathrm{Cs}$ radioactive seeds (loaded into polyglactin suture or mesh).

The LDR dose was $80 \mathrm{~Gy}$ at $0.5 \mathrm{~cm}$ from the implant. Implantation was done with seeds placed $1 \mathrm{~cm}$ apart to achieve the prescribed dose. The HDR dose was $20 \mathrm{~Gy}$ in 2 Gy per fraction, delivered twice daily. High-dose radiation catheters were also placed on the tumor bed $1 \mathrm{~cm}$ apart and planning was done using Brachyvision software (Varian; Palo Alto, CA, USA), and the treatment started within a week of implantation. After securing the brachytherapy catheters/seeds, reconstruction was completed using the previously elevated flap. All pedicled flaps in this series were the pectoralis major muscle (with or without overlying skin), and the free flaps were either rectus abdominus muscle, or anterolateral thigh fascia and overlying skin. A split-thickness skin graft $\left(10-12 / 1000^{\text {th }}\right.$ of an inch) was placed on top of the flap if needed in order to achieve complete wound closure at the completion of the operation.

The primary endpoint of this study was flap viability, defined as survival of the flap sufficient to maintain wound coverage and obviate the need for future procedures in order to obtain would coverage. Secondary endpoints included wound healing complications (classified as minor, which resolved with wound care, and major, which required return to the operating room), seroma, bleeding complications, and surgical site infections. All values are presented as (mean) \pm (standard error of the mean). Statistical analysis was performed using Fisher's exact test (Graphpad Software, Inc; La Jolla, California), and statistical significance was set at $p<0.05$.

\section{Results}

A total of 23 patients were included in this retrospective study; 6 had flap coverage and HDR, and 17 had flap coverage and LDR. The mean age of patients in the HDR group was $57.3 \pm 10.3$ years, and the mean age of patients in the LDR group was $68.9 \pm 13.9$ years (Table 1 ). The diagnosis was recurrent squamous cell carcinoma (SCC) in 21 of 23 subjects $(91.3 \%)$ with recurrent melanoma in one patient who presented with lymph node metastases in the cervical lymph node chains, and one with recurrent mucoepidermoid carcinoma of the submandibular gland. The interval to recurrence was 17.0 months (range 4-72). Seventy-four percent of patients received previous chemotherapy, and all patients had undergone previous radiation therapy with dose range 60-70 Gy in conventional fractionation (Table 1). The type of neck dissection performed by the head and neck surgery service in the 23 patients was a radical neck dissection in 10 patients $(43.5 \%)$, modified radical neck dissection in 6 patients $(26.1 \%)$, and selective neck dissection (some combination of levels $1-4)$ in 6 patients $(26.1 \%)$. Only 1 patient $(4.3 \%)$ did not undergo a formal neck dissection, because he had previously undergone radical neck dissection (Table 1). Pedicled pectoralis major flaps alone were performed in $83.3 \%$ of subjects in the HDR group (5 of 6), and $76.5 \%$ of subjects in the LDR group (13 of 16). Split-thickness
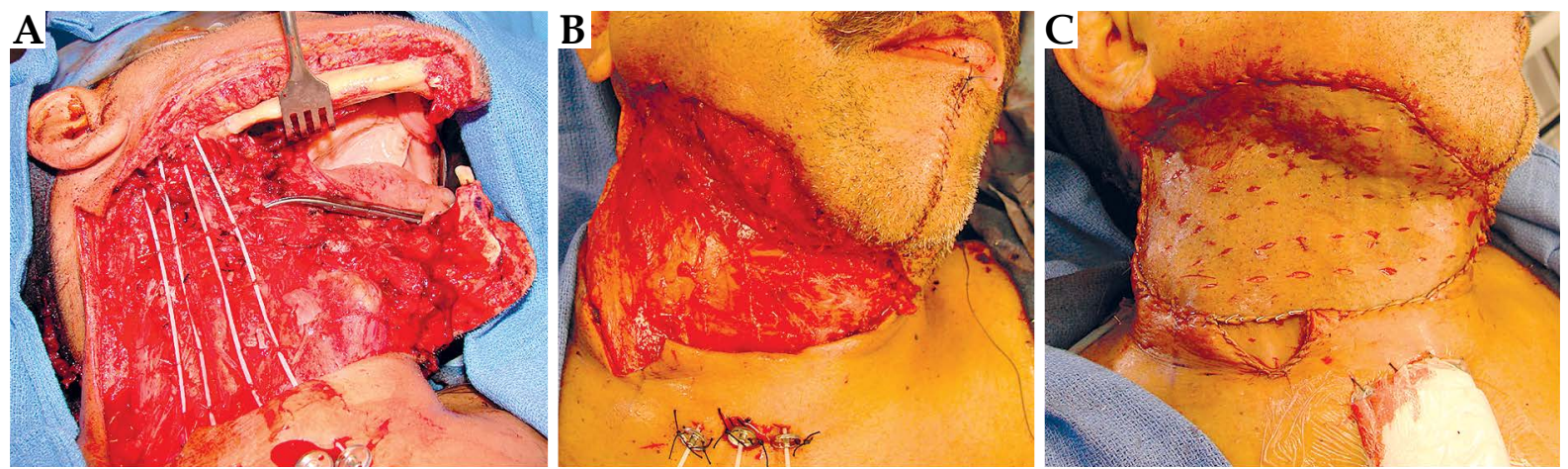

Fig. 1. Intraoperative photographs of A) brachytherapy catheters (HDR) in place, B) catheters covered by pedicled pectoralis major muscle flap (note catheters emerging from skin), and C) overlying split thickness skin graft 


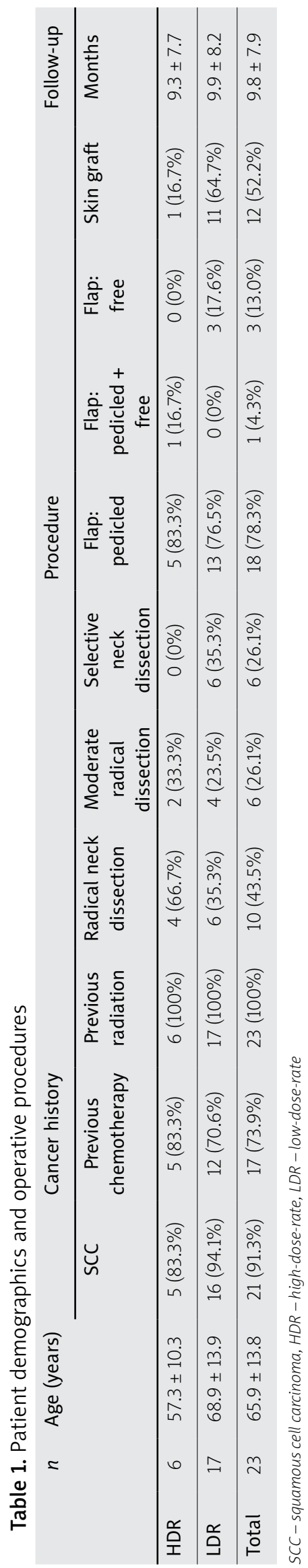

skin grafts harvested from the lateral thigh were performed in $16.7 \%$ (1 of 6) subjects in the HDR group, and $64.7 \%$ (11 of seventeen) subjects in the LDR group (Table 1). Mean follow-up was 9.3 \pm 7.7 months in the HDR group, and $9.9 \pm 8.2$ in the LDR group. In 6 subjects who received HDR, the mean number of ${ }^{192}$ Ir catheters placed was $4.3 \pm 1.2$ (range: $3-6$ ), the mean number of fractions was $9.3 \pm 2.1$ (range: $6-12$ ), and the mean total delivered dose was $21.0 \pm 2.4$ Gy (range: 18-24) (Table 2). In the 17 subjects who received LDR, the mean number of ${ }^{131} \mathrm{Cs}$ seeds was $34.8 \pm 11.7$ (range: 13-55), the mean U/seed was $2.5 \pm 0.3$ (range: $2.0-2.46$ ), and the mean total delivered dose was $81.2 \pm 4.9$ Gy (range: $80-100$ ) (Table 2). Flap viability was $100 \%$ (23 of 23) (Figure 2). There were complications in $50 \%$ of the HDR group ( 3 occurrences of minor wound breakdown that healed with dressings and local wound care), and in $29.4 \%$ of the LDR group ( 2 hematomas, 1 flap infection, and 2 minor wound breakdown that resulted in the development of orocutaneous fistula that resolved with non-operative management) (Table 3 ). Pectoralis major donor site complications occurred in 3 of twenty-two subjects, 2 subjects in the HDR group (1 incisional dehiscence requiring negative pressure dressing, and 1 hematoma requiring evacuation), and 1 subject in the LDR group (incisional eschar that resolved with local wound care). There was no statistically significant difference between the HDR and LDR groups in terms of flap viability or complications $(p>0.05)$.

\section{Discussion}

The concept of local delivery of radiation as a treatment modality nearly immediately followed the discovery of radioactivity by Henri Becquerel in 1896. While the initial iterations of brachytherapy were permanently implanted (and therefore LDR), the development of radiation afterloaders led to the advent of removable catheters (enabling short courses of HDR). This and other innovations in the second half of the twentieth century led to improved patient and healthcare provider safety and efficacy, and therefore a resurgence in interest in brachytherapy.

High-dose and low-dose radiation are important elements of the multi-disciplinary and multi-modality treatment of a number of forms of cancer, including breast, prostate, endometrial, and cervical cancer, in addition to head and neck cancers. Unlike EBR, brachytherapy is able to deliver a high-localized dose with relative sparing of critical normal tissues due to rapid tissue falloff [6], thereby leading to a high rate of local tumor control with limited long-term morbidity $[7,8,9]$. In particular, Pham et al. recently showed that ${ }^{131}$ Cs LDR treatment in RH\&NC led to survival rates comparable to that of EBR, with a reduced rate of radiation-induced toxicity [10]. Similarly, Kishan et al. showed good local recurrence rates and low long-term morbidity with HDR [11].

Almost all patients with RH\&NC who are deemed repeat surgical candidates have been previously treated with EBR. These locally recurrent tumors are usually difficult to resect cleanly, given the extensive tissue changes such as fibrosis, edema, and loss of tissue planes from pri- 
Table 2. Radiation doses

\begin{tabular}{lccccccc} 
& $n$ & Isotope & Catheters & Fractions & Seeds & U/seed & Dose (Gy) \\
\hline HDR & 6 & ${ }^{192} \mathrm{Ir}$ & $4.3 \pm 1.2$ & $9.3 \pm 2.1$ & N/A & N/A & $21.0 \pm 2.4$ \\
\hline LDR & 17 & ${ }^{131} \mathrm{Cs}$ & N/A & N/A & $34.8 \pm 11.7$ & $2.5 \pm 0.3$ & $81.2 \pm 4.9$ \\
\hline Total & 23 & & N/A & N/A & N/A & N/A
\end{tabular}

$H D R$ - high-dose-rate, $L D R$ - low-dose-rate

or EBR. Attaining wide clean margins is difficult in such situations, and this poses a significant risk of local recurrence of tumor despite a complete surgical resection. It is for this reason that brachytherapy is used at our institution in patients with suspicious, close, or positive margins based on frozen section pathological analysis. The quality of the skin and soft tissue within and adjacent to the site of resection and neck dissection site is usually extremely poor. Therefore, in these cases it is nearly impossible to achieve reliable coverage of the resulting wound with primary closure alone. The pectoralis major muscle flap, first described by Ariyan in 1979 [12], remains the most reliable means of providing well-vascularized tissue for coverage of exposed vital structures in the head and neck region. The pectoralis major muscle flap is usually sufficient to provide coverage, but in circumstances where the pectoralis muscle alone is insufficient or unavailable, free tissue transfer is mandated.

While coverage using well-vascularized muscle of this kind is thought to be robust, reliable, and resistant to most local insults, there are rare circumstances when flaps are exposed to conditions as noxious as targeted local radiation. Few reports that exist in the literature that assessed the safety of brachytherapy in combination with flaps suggest that the two modalities can be combined. Ross et al. reviewed their series of patients with head and neck cancer (both primary and recurrent) who were reconstructed using microvascular free tissue transfer, and compared those who had received LDR and those who had not. Their study found that the overall complication rate was higher in the LDR group (38.3\% vs. $15.9 \%$ ) [13]. Despite the higher complication rate, they concluded that the survival benefits conferred by LDR outweighed the manageable increase in complications, and therefore advocated its use. Schiefke et al. retrospectively studied the use of ${ }^{192}$ Ir HDR catheters in 18 patients with either primary or recurrent squamous cell carcinoma, and determined that local HDR did not increase flap morbidity [14]. Moscoso et al. looked at ${ }^{192}$ Ir HDR catheters in 13 subjects with SCC [15], and Panchal et al. looked at ${ }^{192}$ Ir HDR catheters in 10 subjects with SCC, parotid

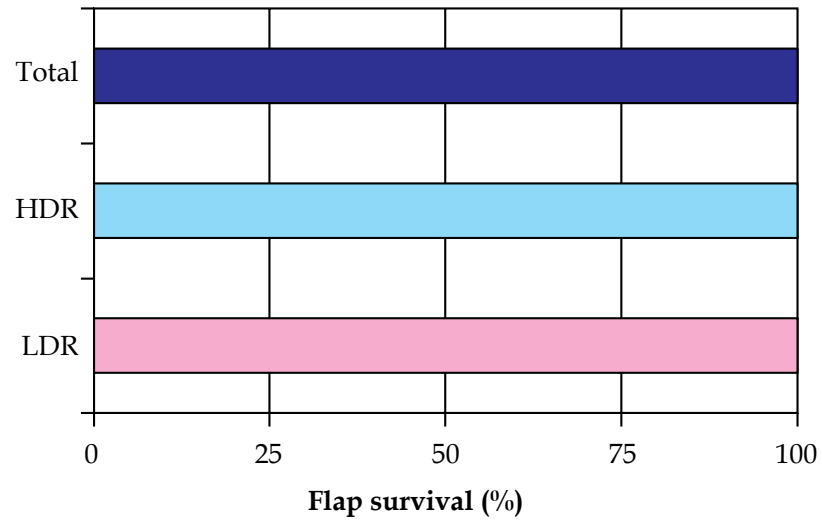

Fig. 2. Flap survival

tumors, or sarcoma [16]. In addition to the heterogeneous histology, the study populations combined subjects with primary and recurrent cancer, and had a mixed population of patients who had undergone prior radiation and those who had not. Those studies concluded that flaps were not compromised by brachytherapy.

These 4 studies make important contributions to the literature, but they leave important questions unanswered including the safety of brachytherapy in the patients with RH\&NC (which is the group at highest risk for complications), and whether there is a difference in healing when patients are treated with HDR versus LDR.

The data presented herein shows 100\% flap viability regardless of the modality of brachytherapy used. Additionally, in this high risk cohort of 23 patients, the overall complication rate was low (only 3 occurrences of minor wound breakdown in the HDR group, and 2 hematomas, 1 superficial surgical site infection, and 2 occurrences of minor wound breakdown in the LDR group). The only complication that required a return to the operating room was the hematoma, which was drained. The patient went on to heal uneventfully.

Taken together, the results of our study indicate that either HDR or LDR can be safely delivered with con-

Table 3. Flap complications

\begin{tabular}{lccccccc} 
& $n$ & Hematoma & Infection & Seroma & $\begin{array}{c}\text { Minor wound } \\
\text { beakdown }\end{array}$ & $\begin{array}{c}\text { Major wound } \\
\text { breakdown }\end{array}$ & $\begin{array}{c}\text { Total flap } \\
\text { complications }\end{array}$ \\
\hline HDR & 6 & $0(0 \%)$ & $0(0 \%)$ & $0(0 \%)$ & $3(50 \%)$ & $0(0 \%)$ & $3(50 \%)$ \\
\hline LDR & 17 & $2(11.8 \%)$ & $1(5.9 \%)$ & $0(0 \%)$ & $2(11.8 \%)$ & $0(0 \%)$ & $5(29.4 \%)$ \\
\hline Total & 23 & $2(8.7 \%)$ & $1(4.3 \%)$ & $0(0 \%)$ & $5(21.7 \%)$ & $0(0 \%)$ & $8(34.8 \%)$
\end{tabular}

$H D R$ - high-dose-rate, $L D R$ - low-dose-rate 
comitant flap reconstruction. The data does not indicate that either form of brachytherapy is more likely to cause flap morbidity (although this finding may be limited by the relatively small number of patients included in the study), and therefore one form of brachytherapy cannot be recommended over the other.

\section{Conclusions}

In conclusion, in patients who have flap reconstruction and immediate postoperative radiotherapy following salvage procedures for recurrent head and neck cancer, neither HDR nor LDR impacted long term flap viability or skin graft survival. Flap coverage of defects (with or without skin grafting) in combination with brachytherapy is a safe and effective means of providing soft tissue coverage in these challenging patients.

\section{Disclosure}

Authors report no conflict of interest.

\section{References}

1. Brockstein B, Haraf DJ, Rademaker AW et al. Patterns of failure prognostic factors and survival in locoregionally advanced head and neck cancer treated with concomitant chemoradiotherapy: a 9-year, 337-patient, multi-institutional experience. Ann Oncol 2004; 15: 1179-1186.

2. Rudzianskas V, Inciura Z, Juozaityte E et al. Reirradiation of recurrent head and neck cancer using high-dose-rate brachytherapy. Acta Otorhinolaryngol Ital 2012; 32: 297-303.

3. Shasha D, Harrison LB, Chiu-Tsao ST. The role of brachytherapy in head and neck cancer. Semin Radiat Oncol 1998; 8: 270-281.

4. Righi PD, Weisberger EC, Krakovits PR et al. Wound complications associated with brachytherapy for primary or salvage treatment of head and neck cancer. Laryngoscope 1997; 107: 1464-1468.

5. Park RL, Liberman FZ, Lee DJ et al. Iodine-125 seed implantation as an adjunct to surgery in advanced recurrent squamous cell cancer of the head and neck. Laryngoscope 1991; 101: 405-410

6. Nag S, Cano ER, Demanes DJ et al. The American Brachytherapy Society recommendations for high-dose-rate brachytherapy for head-and-neck carcinoma. Int J Radiat Oncol Biol Phys 2001; 50: 1190-1198.

7. Hepel JT, Syed AM, Puthawala A et al. Salvage high-doserate (HDR) brachytherapy for recurrent head-and-neck cancer. Int J Radiat Oncol Biol Phys 2005; 62: 1444-1450.

8. Grimard L, Esche B, Lamothe A et al. Interstitial low-doserate brachytherapy in the treatment of recurrent head and neck malignancies. Head Neck 2006; 28: 888-895.

9. Bollet MA, Lapeyre M, Marchal C et al. Cervical lymph node relapses of head-and-neck squamous cell carcinoma: is brachytherapy a therapeutic option? Int J Radiat Oncol Biol Phys 2001; 51: 1305-1312.

10. Pham A, Arora S, Wernicke G et al. Cesium-131 brachytherapy in high risk and recurrent head and neck cancers: first report of long-term outcomes. J Contemp Brachytherapy 2015; 7: 445-452.

11. Kishan AU, Lee EW, McWilliams J et al. Image-guided highdose rate brachytherapy: preliminary outcomes and toxicity of a joint interventional radiology and radiation oncology technique for achieving local control in challenging cases. J Contemp Brachytherapy 2015; 7: 327-335.
12. Ariyan S. The pectoralis major myocutaneous flap. A versatile flap for reconstruction in the head and neck. Plast Reconstr Surg 1979; 63: 73-81.

13. Ross DA, Hundal JS, Son YH et al. Microsurgical free flap reconstruction outcomes in head and neck cancer patients after surgical extirpation and intraoperative brachytherapy. Laryngoscope 2004; 114: 1170-1176.

14. Schiefke F, Hildebrandt G, Pohlmann S et al. Combination of surgical resection and HDR-brachytherapy in patients with recurrent or advanced head and neck carcinomas. J Craniomaxillofac Surg 2008; 36: 285-292.

15. Moscoso JF, Urken ML, Dalton J et al. Simultaneous interstitial radiotherapy with regional or free-flap reconstruction, following salvage surgery of recurrent head and neck carcinoma: Analysis of complications. Arch Otolaryngol Head Neck Surg 1994; 120: 965-972.

16. Panchal JI, Agrawal RK, McLean NR et al. Early postoperative brachytherapy following free flap reconstruction. $\mathrm{Br} J$ Plast Surg 1993; 46: 511-515. 